SISSA Preprint 78/2010/fm

arXiv: 1011.1003

\title{
PICARD GROUP OF HYPERSURFACES IN TORIC 3-FOLDS
}

\author{
UGO BRUZZO $\S_{\S}$ AND ANTONELLA GRASSI \\ ฯ Department of Mathematics, University of Pennsylvania, \\ David Rittenhouse Laboratory, 209 S 33rd Street, \\ Philadelphia, PA 19104, USA \\ $\S$ Istituto Nazionale di Fisica Nucleare, Sezione di Trieste
}

\begin{abstract}
We show that the usual sufficient criterion for a very general hypersurface in a smooth projective manifold to have the same Picard number as the ambient variety can be generalized to quasi-smooth hypersurfaces in complete simplicial toric varieties. This sufficient condition always holds for very general K3 surfaces embedded in Fano toric 3-folds.
\end{abstract}

Date: October 25, 2018.

1991 Mathematics Subject Classification. 14C22,14J70, 14M25.

E-mail: bruzzo@math.upenn.edu, bruzzo@sissa.it, grassi@sas.upenn.edu.

$\dagger$ On leave of absence from Scuola Internazionale Superiore di Studi Avanzati, Via Bonomea 265,34136 Trieste, Italy

Support for this work was provided by the NSF Research Training Group Grant DMS-0636606, by PRIN "Geometria delle varietà algebriche e dei loro spazi dei moduli " and the INFN project PI14 "Nonperturbative dynamics of gauge theories". U.B. is a member of the VBAC group. 


\section{InTRODUCTION}

In this paper we study the Noether-Lefschetz problem for hypersurfaces in complete simplicial toric threefolds, namely, we prove that under a certain condition, a very general hypersurface in an ample linear system in such a toric threefold $\mathbb{P}_{\Sigma}$ has the same Picard number as $\mathbb{P}_{\Sigma}$. In particular, this holds for a very general K3 hypersurface in the anticanonical system of a simplicial toric Fano threefold. (A property is very general if it holds in the complement of countably many proper closed subvarieties [16].)

This result can be regarded on one hand as a first step towards the study of Noether-Lefschetz loci of the moduli space of $K 3$ hypersurfaces in a toric Fano threefolds; see also the recent works of [14, 17, 15]. On the other hand, this completes the picture for computing the Picard number for certain hypersurfaces in the anticanonical system of a toric Fano variety, by handling the unknown case in dimension 3 .

Recall that the Picard number, $\rho(Y)$ of a variety $Y$ is the rank of the NéronSeveri group, that is of the image of the Picard group in the second cohomology group with integer coefficients. The Picard group and the Picard number of a toric variety $\mathbb{P}_{\Sigma}$ can be easily computed from the combinatorial data of $\Sigma$. Let $X$ be a nondegenerate hypersurface in the anticanonical system of a simplicial toric Fano variety $\mathbb{P}_{\Sigma}$, with $\operatorname{dim} \mathbb{P}_{\Sigma} \geq 4$ (note that a general hypersurface is also nondegenerate). In the 80s and $90 \mathrm{~s}$ it was shown [8, 2, 10, 1], that the Picard number of any such $X$ can be explicitly computed from combinatorial data. This result was a pivotal ingredient in describing the toric version of mirror symmetry (see for example [8]). The argument in the above papers is essentially topological and computes the dimension of the second cohomology group of $X$, which happens to be equal to $\rho(X)$ if $\operatorname{dim}(X) \geq 3$, but not necessarily if $\operatorname{dim}(X)=2$. In addition, even the statement in the above papers does not hold when $\operatorname{dim} \mathbb{P}_{\Sigma}=3$, as we see from the case of Fermat's quartic in $\mathbb{P}^{3}$, which is nondegenerate.

This type of result was generalized by Roan to the case of toric varieties (not necessarily Fano) also for the case when the ambient variety has dimension $d \geq$ 4 [19], and by Ravindra and Srinivas to general normal varieties, still with the restriction $d \geq 4$ [18].

This paper then fills the gap for $\operatorname{dim} \mathbb{P}_{\Sigma}=3$. It was already known that $\rho(X)=\rho\left(\mathbb{P}_{\Sigma}\right)$ for particular cases of toric Fano threefolds, namely certain weighted projective spaces [5, 24, 11], as in the higher dimensional case. The techniques used in the case of weighted projective spaces are very much tailored to that specific case 
[12, 24, 11]. On the other hand, the classical infinitesimal techniques introduced in the 70s by Griffiths, Steenbrink and collaborators to solve the Noether-Lefschetz problem in the smooth case (see for example [4]) cannot be used due to the presence of singularities. Our argument is partly inspired by Cox's paper [5]: it generalizes the classical infinitesimal techniques and combines them with more recent results about toric varieties, their Cox ring and their cohomology [3]. In fact, $X$ and $\mathbb{P}_{\Sigma}$ are projective orbifolds, and a pure Hodge structure can be defined for them; this will be a key tool in the proof.

In Section 2 we mostly recall some relevant results from [3], and adapt them to the set up of [4]. We start with basic properties of simplicial toric varieties and general hypersurfaces defined by ample divisors. Moreover we note that the exact sequence defining the primitive cohomology in middle dimension of such a hypersurface splits orthogonally with respect to the intersection pairing. The middle cohomology is the sum of the primitive cohomology and the "fixed" cohomology, i.e., the cohomology inherited from the ambient toric variety; the splitting is consistent with the Hodge decomposition. We then state some results of [3] which express the primitive cohomology in middle degree in terms of the Jacobian ring of the hypersurface; here we assume that ambient space has odd dimension.

Section 3 contains the bulk of the argument: we proceed along the lines of the infinitesimal arguments of Griffiths for smooth varieties and adapt it to the toric case. We start from the moduli space of quasi-smooth hypersurfaces constructed in [3], consider a natural Gauss-Manin connection, proceed to prove an infinitesimal Noether-Lefschetz theorem and then the needed global Noether-Lefschetz theorem. Finally, we focus on the case of $K 3$ hypersurfaces in the anticanonical system of a simplicial toric Fano threefold.

The suggestion that a very general hypersurface in a toric Fano threefold $\mathbb{P}_{\Sigma}$ has the same Picard number as the ambient variety can be found, in a different language, in an unpublished paper by Rohsiepe [20] (see the formula and Remark in the middle of page 3), based on some dimension counting arguments and trying to generalize to the case $\operatorname{dim} \mathbb{P}_{\Sigma}=3$ a formula that Batyrev proved for $\operatorname{dim} \mathbb{P}_{\Sigma}=4$ [2].

Acknowledgements. We thank Eduardo Cattani, Alberto Collino, David Cox, Igor Dolgachev, Luca Migliorini, Vittorio Perduca, Domingo Toledo and the referee for useful discussions and suggestions. We are grateful for the hospitality and support offered by the University of Pennsylvania and SISSA. The first author would also like to thank the staff and the scientists at Penn's Department of Mathematics for providing an enjoyable and productive atmosphere. 


\section{Hypersurfaces in SIMPliCiAL COMPlEte TORIC VARIETIES}

In this section we recall some basic facts about hypersurfaces in toric varieties and their cohomology. We mainly follow the notation in [3]. All schemes are schemes over the complex numbers.

2.1. Preliminaries and notation. Let $M$ be a free abelian group of rank $d$, let $N=\operatorname{Hom}(M, \mathbb{Z})$, and $N_{\mathbb{R}}=N \otimes_{\mathbb{Z}} \mathbb{R}$.

Definition 2.1. [3, Def. 1.1 and 1.3]

(i) A convex subset $\sigma \subset N_{\mathbb{R}}$ is a rational $k$-dimensional simplicial cone if there exist $k$ linearly independent primitive elements $e_{1}, \ldots, e_{k} \in N$ such that $\sigma=\left\{\mu_{1} e_{1}+\cdots+\mu_{k} e_{k}\right\}$, with $\mu_{i}$ nonnegative real numbers. The generators $e_{i}$ are said to be integral if for every $i$ and any nonnegative rational number $\mu$, the product $\mu e_{i}$ is in $N$ only if $\mu$ is an integer.

(ii) Given two rational simplicial cones $\sigma, \sigma^{\prime}$, one says that $\sigma^{\prime}$ is a face of $\sigma$ (we then write $\sigma^{\prime}<\sigma$ ) if the set of integral generators of $\sigma^{\prime}$ is a subset of the set of integral generators of $\sigma$.

(iii) A finite set $\Sigma=\left\{\sigma_{1}, \ldots, \sigma_{s}\right\}$ of rational simplicial cones is called a rational simplicial complete d-dimensional fan if

(a) all faces of cones in $\Sigma$ are in $\Sigma$;

(b) if $\sigma, \sigma^{\prime} \in \Sigma$, then $\sigma \cap \sigma^{\prime}<\sigma$ and $\sigma \cap \sigma^{\prime}<\sigma^{\prime}$;

(c) $N_{\mathbb{R}}=\sigma_{1} \cup \cdots \cup \sigma_{s}$.

A rational simplicial complete $d$-dimensional fan $\Sigma$ defines a toric variety $\mathbb{P}_{\Sigma}$ of dimension $d$ having only Abelian quotient singularities. Moreover, $\mathbb{P}_{\Sigma}$ is simply connected, and is an orbifold. We shall use the term "orbifold" in the following sense (see, e.g., [8], Def. A.2.1): an $n$-dimensional variety $Y$ is an orbifold if every point $y \in Y$ has a neighborhood which is isomorphic to $U / G$ as an analytic space, where $G$ is a subgroup of $G l_{n}(\mathbb{C})$ with no nontrivial complex reflections, and $U$ is a $G$-invariant neighborhood of the origin of $\mathbb{C}^{n}$. (A complex reflection is an element in $G l_{n}(\mathbb{C})$ with $n-1$ eigenvalues equal to 1.) A sub-orbifold of an orbifold $Y$ is a subvariety $Y^{\prime} \subset Y$ with the property that for every $y \in Y^{\prime}$ there is a local chart $(U / G, 0)$ of $Y$ at $y$ such that the inverse image of $Y^{\prime}$ in $U$ is smooth at 0 . Intuitively, a sub-orbifold is a subvariety whose only singularities come from the ambient variety. These notions of orbifold and sub-orbifold are synonymous to those of $V$-manifold and sub- $V$-manifold, which is indeed the terminology used in [3]. The notion of $V$-manifold is originally due to Satake [22]. 
Definition/Proposition 2.2. Let $C l(\Sigma)$ be the group of Weil divisors in $\mathbb{P}_{\Sigma}$ modulo rational equivalence, and let $\operatorname{Pic}(\Sigma)$ be the group of line bundles on $\mathbb{P}_{\Sigma}$ modulo isomorphism. As the notation suggests, both are intrinsic to the fan $\Sigma$. Both are finitely generated Abelian groups, and $\mathrm{Pic}(\Sigma)$ is actually free. Moveover, under our assumptions the toric variety $\mathbb{P}_{\Sigma}$ is $\mathbb{Q}$-factorial, i.e., the natural inclusion $\operatorname{Pic}(\Sigma) \hookrightarrow C l(\Sigma)$ becomes an isomorphism if one tensors by $\mathbb{Q}$. The rank of the two groups, denoted by $\rho(\Sigma)$, is also the Picard number, the rank of the Néron-Severi group of $\mathbb{P}_{\Sigma}$.

Recall that the Néron-Severi group of a variety $Y$ is the image of the Picard group in the second cohomology group with integer coefficients. One can define its $\operatorname{rank}$ as $\rho(Y) \stackrel{\text { def }}{=} \operatorname{dim}_{\mathbb{Q}} N S(Y) \otimes_{\mathbb{Z}} \mathbb{Q}=\operatorname{dim}_{\mathbb{Q}} H^{2}(Y, \mathbb{Q}) \cap H^{1,1}(Y, \mathbb{C})$.

The group $\mathbf{D}(\Sigma)=$ Spec $\mathbb{C}[C l(\Sigma)]$ is an affine algebraic group whose character group is isomorphic to $C l(\Sigma)$. Since there is a surjection $\mathbb{Z}^{n} \rightarrow C l(\Sigma)$, we have an embedding $\mathbf{D}(\Sigma) \hookrightarrow\left(\mathbb{C}^{*}\right)^{n}$, and a natural action of $\mathbf{D}(\Sigma)$ on the affine space $\mathbb{A}^{n}$. The quotient $\mathbf{T}(\Sigma)=\left(\mathbb{C}^{*}\right)^{n} / \mathbf{D}(\Sigma)$ is an algebraic torus. Below we shall show that this group acts naturally on $\mathbb{P}_{\Sigma}$.

Definition 2.3. ([6] Given a fan $\Sigma$, consider a variable $z_{i}$ for each 1-dimensional cone $\varsigma_{i}$ in $\Sigma$, and let $S(\Sigma)$ be the polynomial ring $\mathbb{C}\left[z_{1}, \ldots, z_{n}\right]$. For every $\sigma \in \Sigma$, let $z_{\sigma}=\prod_{\varsigma_{i} \not \subset \sigma} z_{i}$, and let $B(\Sigma)$ the ideal in $S(\Sigma)$ generated by the $z_{\sigma}$ 's.

$S(\Sigma)$ is called the Cox ring.

$S(\Sigma)$ is a graded ring, with grading provided by the class group, $S(\Sigma)=$ $\oplus_{\beta \in C l(\Sigma)} S_{\beta}$. We identify the affine space $\mathbb{A}^{n}$ with Spec $S(\Sigma)$, and denote by $Z(\Sigma)$ the affine variety in $\mathbb{A}^{n}$ given by the ideal $B(\Sigma)$. If we set $U(\Sigma)=\mathbb{A}^{n}-Z(\Sigma)$, the group $\mathbf{D}(\Sigma)$ acts on $U(\Sigma)$, and the toric variety $\mathbb{P}_{\Sigma}$ may be represented as $U(\Sigma) / \mathbf{D}(\Sigma)$. This yields an action of $\mathbf{T}(\Sigma)$ on $\mathbb{P}_{\Sigma}$. For every face $\tau$ in $\Sigma$ we shall denote by $\mathbf{T}_{\tau} \subset \mathbb{P}_{\Sigma}$ the orbit of $\tau$ in $\mathbb{P}_{\Sigma}$ under this action.

2.2. Quasi-smooth hypersurfaces. From now on we assume that $\mathbb{P}_{\Sigma}$ is projective. Let $L$ be an ample line bundle on $\mathbb{P}_{\Sigma}$, and denote by $\beta \in C l(\Sigma)$ its degree; a section of $L$ is a polynomial in $S_{\beta}$.

Definition 2.4. [3, Def. 3.1] Let $f$ be a section of $L$, and let $\mathbf{V}(f)$ be the zero locus of $f$ in $\operatorname{Spec} S(\Sigma)$. We say that the hypersurface $X$ cut in $\mathbb{P}_{\Sigma}$ by the equation $f=0$ is quasi-smooth if $\mathbf{V}(f)$ is smooth outside $Z(\Sigma)$. 
Definition 2.5. [3, Def. 4.13] If $L$ is an ample line bundle on $\mathbb{P}_{\Sigma}$, a hypersurface $X$ is said to be nondegenerate if $X \cap \mathbf{T}_{\tau}$ is a smooth 1-codimensional subvariety of $\mathbf{T}_{\tau}$ for all $\tau$ in $\Sigma$.

Proposition 2.6. [3, Prop. 3.5, 4.15] If $f$ is the general section of an ample invertible sheaf, then $X$ is nondegenerate. Moreover, every nondegenerate hypersurface $X \subset \mathbb{P}_{\Sigma}$ is quasi-smooth. Thus, if $f$ is a general section of $L$, its zero locus is a quasi-smooth hypersurface $X$ in $\mathbb{P}_{\Sigma}$, hence it is an orbifold.

An important fact is that the complex cohomology of an orbifold has a pure Hodge structure in each dimension [23, 25].

We also note that in view of the homotopy hyperplane Lefschetz theorem, which holds for the embedding $X \hookrightarrow \mathbb{P}_{\Sigma}$ [13, Thm. 1.2 Part II], $X$ is simply connected if $\operatorname{dim}\left(\mathbb{P}_{\Sigma}\right) \geq 3$

2.3. Primitive cohomology of a hypersurface. Let $L$ be an ample line bundle on $\mathbb{P}_{\Sigma}$, and let $X$ be a hypersurface in $\mathbb{P}_{\Sigma}$ cut by a section $f$ of $L$ (note that by [3], Proposition 10.8, $f$ lies in $B(\Sigma)$ ). Denote by $i: X \rightarrow \mathbb{P}_{\Sigma}$ the inclusion, and by $i^{*}: H^{\bullet}\left(\mathbb{P}_{\Sigma}, \mathbb{C}\right) \rightarrow H^{\bullet}(X, \mathbb{C})$ the associated morphism in cohomology; $i^{*}: H^{d-1}\left(\mathbb{P}_{\Sigma}, \mathbb{C}\right) \rightarrow H^{d-1}(X, \mathbb{C})$ is injective by Proposition 10.8 in [3].

Definition 2.7. [3, Def. 10.9] The primitive cohomology group $\mathrm{PH}^{d-1}(X)$ is the quotient $H^{d-1}(X, \mathbb{C}) / i^{*}\left(H^{d-1}\left(\mathbb{P}_{\Sigma}, \mathbb{C}\right)\right)$.

Lemma 2.8. The exact sequence

$$
0 \rightarrow i^{*}\left(H^{d-1}\left(\mathbb{P}_{\Sigma}, \mathbb{C}\right)\right) \rightarrow H^{d-1}(X, \mathbb{C}) \rightarrow P H^{d-1}(X) \rightarrow 0
$$

splits orthogonally with respect to the intersection pairing in $H^{\bullet}(X, \mathbb{C})$. The same is true with coefficients in $\mathbb{Q}$.

Proof. The hard Lefschetz theorem holds also for projective orbifolds (this follows from the results in [21]; a simple proof is given in [26]).

Then cupping by $c_{1}(L)$ we get an isomorphism $\ell: H^{d-1}\left(\mathbb{P}_{\Sigma}, \mathbb{C}\right) \rightarrow H^{d+1}\left(\mathbb{P}_{\Sigma}, \mathbb{C}\right)$. Let $i_{*}: H^{d-1}(X, \mathbb{C}) \rightarrow H^{d+1}\left(\mathbb{P}_{\Sigma}, \mathbb{C}\right)$ be the Gysin map. We claim that the following 
commutative diagram

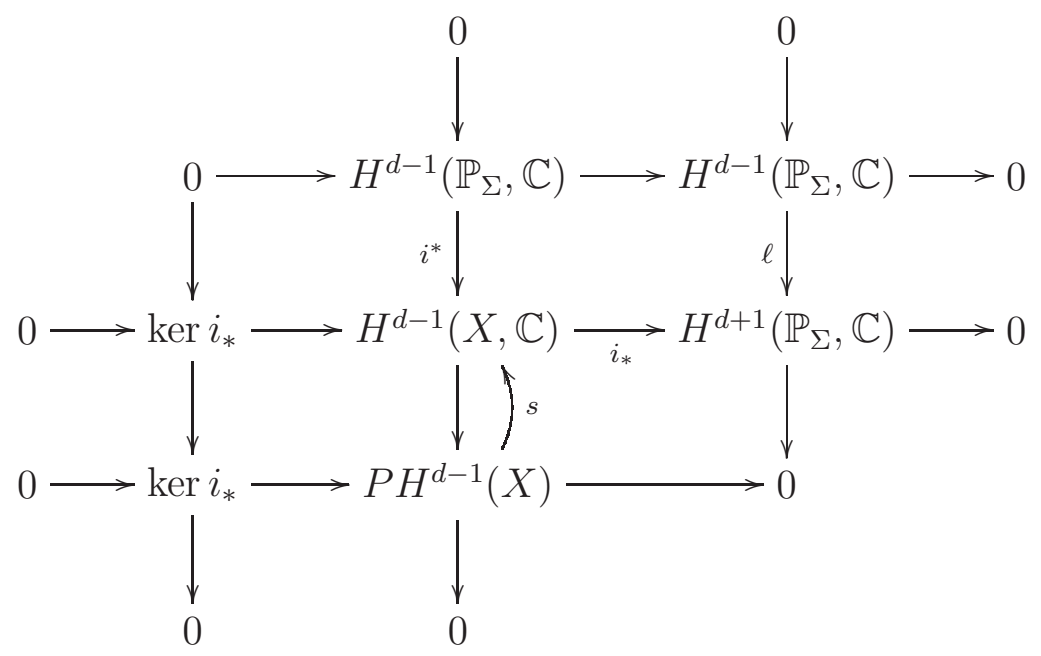

provides a straightforward splitting $s$ of the above exact sequence. Let $\langle$,$\rangle be the$ intersection pairing in cohomology both in $H^{\bullet}(X, \mathbb{C})$ and $H^{\bullet}\left(\mathbb{P}_{\Sigma}, \mathbb{C}\right)$, and recall that $i^{*}$ and $i_{*}$ are adjoint with respect to the intersection pairing. The upper-right square commutes since by Poincaré duality

$$
\left\langle i_{*} i^{*} \alpha, \beta\right\rangle=\left\langle i^{*} \alpha, i^{*} \beta\right\rangle=\left\langle c_{1}(L) \cup \alpha, \beta\right\rangle=\langle\ell(\alpha), \beta\rangle .
$$

If $\alpha \in H^{d-1}\left(\mathbb{P}_{\Sigma}, \mathbb{C}\right)$ and $\beta \in P H^{d-1}(X)$, we have

$$
\left\langle i^{*}(\alpha), s(\beta)\right\rangle=\left\langle\alpha, i_{*}(s(\beta))\right\rangle=0 .
$$

If the statement is true with coefficients in $\mathbb{C}$ it also true with coefficients in $\mathbb{Q}$ since $H^{\bullet}(X, \mathbb{C}) \simeq H^{\bullet}(X, \mathbb{Q}) \otimes_{\mathbb{Q}} \mathbb{C}$.

Remark 2.9. The kernel of $i_{*}$ in $H^{d-1}(X, \mathbb{C})$ is sometimes called the "variable cohomology" $H_{\text {var }}^{d-1}(X, \mathbb{C})$; in degree $d-1$ the variable and primitive cohomologies of $X$ are then isomorphic.

Both $H^{d-1}\left(\mathbb{P}_{\Sigma}, \mathbb{C}\right)$ and $H^{d-1}(X, \mathbb{C})$ have pure Hodge structures, and the morphism $i^{*}$ is compatible with them, so that $P H^{d-1}(X)$ inherits a pure Hodge structure. We shall write

$$
P H^{d-1}(X)=\bigoplus_{p=0}^{d-1} P H^{p, d-1-p}(X) .
$$

The following Proposition 2.10 implicitly uses a generalization of Bott's vanishing theorem, called the Bott-Steenbrink-Danilov theorem, which indeed holds under our assumptions. The exact statement is that $H^{i}\left(\mathbb{P}_{\Sigma}, \Omega_{\mathbb{P}_{\Sigma}}^{p}(L)\right)=0$ for all 
$i>0$ and $p \geq 0$ if $L$ is an ample line bundle on $\mathbb{P}_{\Sigma}$. This was stated without proof by Danilov [9] and proved in [3] (Theorem 7.1).

Proposition 2.10. There is a natural isomorphism

$$
P H^{p, d-p-1}(X) \simeq \frac{H^{0}\left(\mathbb{P}_{\Sigma}, \Omega_{\mathbb{P}_{\Sigma}}^{d}((d-p+1) X)\right.}{H^{0}\left(\mathbb{P}_{\Sigma}, \Omega_{\mathbb{P}_{\Sigma}}^{d}((d-p) X)+d H^{0}\left(\mathbb{P}_{\Sigma}, \Omega_{\mathbb{P}_{\Sigma}}^{d-1}((d-p) X)\right.\right.}
$$

Proof. This follows from comparing Corollaries 10.2 and 10.12 in [3].

The resulting projection map, multiplied by the factor $(-1)^{p-1} /(d-p+1)$ !, will be denoted by

$$
r_{p}: H^{0}\left(\mathbb{P}_{\Sigma}, \Omega_{\mathbb{P}_{\Sigma}}^{d}((d-p+1) X) \rightarrow P H^{p, d-p-1}(X)\right.
$$

and is called the $p$-th residue map in analogy with the classical case.

Definition 2.11. Let $X$ be any hypersurface in $\mathbb{P}_{\Sigma}$ cut by a section $f$ of $L$ and let $J(f)$ be the ideal of the Cox ring generated by the derivatives of $f$. The ring $R(f)=S(\Sigma) / J(f)$ is the Jacobian ring of $S(\Sigma)$.

The Jacobian ring encodes all the information about the primitive cohomology of $X$ :

Proposition 2.12. If $p \neq d / 2-1, P H^{p, d-p-1}(X) \simeq R(f)_{(d-p) \beta-\beta_{0}}$, where $\beta_{0}=$ $-\operatorname{deg} K_{\mathbb{P}_{\Sigma}}, \beta=\operatorname{deg} L$.

Proof. [3] Theorem 10.13.

\section{The Picard group of the General toric threefold}

3.1. The Gauss-Manin connection. Let $z$ be the open subscheme of $|L|$ parametrizing the quasi-smooth hypersurfaces in $|L|$, and let $\pi: \mathscr{F} \rightarrow z$ be the tautological family on $z$; we denote by $X_{z}$ the fiber of $\mathscr{F}$ at $z \in z$. Let $\mathscr{H}^{d-1}$ be the local system on $Z$ whose fiber at $z$ is the cohomology $H^{d-1}\left(X_{z}\right)$, i.e., $\mathscr{H}^{d-1}=$ $R^{d-1} \pi_{*} \mathbb{C}$. It defines a flat connection $\nabla$ in the vector bundle $\mathscr{E}^{d-1}=\mathscr{H}^{d-1} \otimes_{\mathbb{C}} \mathcal{O}_{z}$, the Gauss-Manin connection of $\mathscr{E}^{-1}$. Since the hypersurfaces $X_{z}$ are quasi-smooth, the Hodge structure of the fibres $H^{d-1}\left(X_{z}\right)$ of $\mathscr{E}^{d-1}$ varies analytically with $z$ [23. The corresponding filtration defines holomorphic subbundles $F^{p} \mathscr{E}^{d-1}$, and the graded object of the filtration defines holomophic bundles $G r_{F}^{p}\left(\mathscr{E}^{d-1}\right)$. The bundles $\mathscr{E} p, d-p-1$ given by the Hodge decomposition are not holomorphic subbundles of $\mathscr{E}^{d-1}$, but are diffeomorphic to $G r_{F}^{p}\left(\mathscr{E}^{d-1}\right)$, and as such they have a holomorphic 
structure. The quotient bundles $\mathscr{P} \mathscr{E}^{p, d-p-1}$ of $\mathscr{E}^{p, d-p-1}$ correspond to the primitive cohomologies of the hypersurfaces $X_{z}$. Let $\pi_{p}: \mathscr{E}^{d-1} \rightarrow \mathscr{P} \mathscr{E}^{p, d-p+1}$ be the natural projection.

We denote by $\tilde{\gamma}_{p}$ the cup product

$$
\tilde{\gamma}_{p}: H^{0}\left(\mathbb{P}_{\Sigma}, \mathcal{O}_{\mathbb{P}_{\Sigma}}(X)\right) \otimes H^{0}\left(\mathbb{P}_{\Sigma}, \Omega_{\mathbb{P}_{\Sigma}}^{d}((d-p) X)\right) \rightarrow H^{0}\left(\mathbb{P}_{\Sigma}, \Omega_{\mathbb{P}_{\Sigma}}^{d}((d-p+1) X)\right) .
$$

If $z_{0}$ is the point in $Z$ corresponding to $X$, the space $H^{0}\left(\mathbb{P}_{\Sigma}, \mathcal{O}_{\mathbb{P}_{\Sigma}}(X)\right) / \mathbb{C}(f)$, where $\mathbb{C}(f)$ is the 1-dimensional subspace of $H^{0}\left(\mathbb{P}_{\Sigma}, \mathcal{O}_{\mathbb{P}_{\Sigma}}(X)\right)$ generated by $f$, can be identified with $T_{z_{0}} z$. The morphism $\tilde{\gamma}_{p}$ induces in cohomology the Gauss-Manin connection:

Lemma 3.1. Let $\sigma_{0}$ be a primitive class in $P H^{p, d-p-1}(X)$, let $v \in T_{z_{0}} Z$, and let $\sigma$ be a section of $\mathscr{E}^{p, d-p-1}$ along a curve in $\mathcal{Z}$ whose tangent vector at $z_{0}$ is $v$, such that $\sigma\left(z_{0}\right)=\sigma_{0}$.

Then

$$
\pi_{p-1}\left(\nabla_{v}(\sigma)\right)=r_{p-1}\left(\tilde{\gamma}_{p}(\tilde{v} \otimes \tilde{\sigma})\right)
$$

where $r_{p}, r_{p-1}$ are the residue morphisms defined in equation (1), $\tilde{\sigma}$ is an element in $H^{0}\left(\mathbb{P}_{\Sigma}, \Omega_{\mathbb{P}_{\Sigma}}^{d}((d-p+1) X)\right)$ such that $r_{p}(\tilde{\sigma})=\sigma_{0}$, and $\tilde{v}$ is a pre-image of $v$ in $H^{0}\left(\mathbb{P}_{\Sigma}, \mathcal{O}_{\mathbb{P}_{\Sigma}}(X)\right)$.

In particular the following diagram commutes:

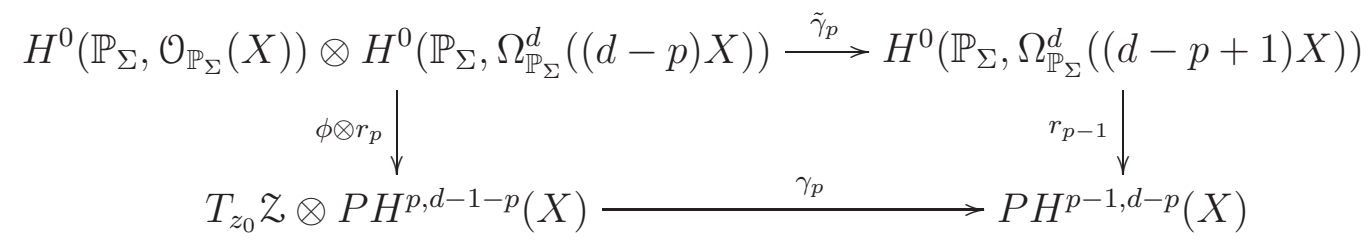

where $\gamma_{p}$ is the morphism that maps $v \otimes \alpha$ to $\nabla_{v} \alpha$ and $\phi$ is the projection $\phi:$ $H^{0}\left(\mathbb{P}_{\Sigma}, \mathcal{O}_{\mathbb{P}_{\Sigma}}(X)\right) \rightarrow T_{z_{0}} z$.

Proof. This is a standard computation, see [4], Proposition 5.4.3. Let $f_{i}$ be local representatives, with respect to a suitable cover $\left\{U_{i}\right\}$ of $\mathbb{P}_{\Sigma}$, of the section $f$. Via the isomorphism of Proposition 2.10, we locally represent $\sigma_{0}$ by the meromorphic differential forms $\omega_{i} / f_{i}^{d-p+1}$. A tangent vector $v \in T_{z_{0}} z$ represents a deformation $f_{i} \mapsto f_{i}+t g_{i}$ where $t$ is a complex parameter, and $g_{i}$ are holomorphic functions. Then $\nabla_{v}(\sigma)$ is represented by

$$
\left[\frac{d}{d t} \frac{\omega_{i}}{\left(f_{i}+t g_{i}\right)^{d-p+1}}\right]_{t=0}=-(d-p+1) \frac{g_{i} \omega_{i}}{f_{i}^{d-p+2}} .
$$


But the right-hand side of this equation is, up to a suitable factor, the argument of the map $r_{p-1}$ in the right-hand side of equation (2).

Lemma 3.2. If $\alpha$ and $\eta$ are sections of $\mathscr{E}^{p, d-p-1}$ and $\mathscr{E}^{d-p, p-1}$ respectively, then for every tangent vector $v \in T_{z_{0}} z$,

$$
\nabla_{v} \alpha \cup \eta=-\alpha \cup \nabla_{v} \eta
$$

Proof. The Gauss-Manin connection is compatible with the cup product by definition, i.e.,

$$
\nabla_{v}(\alpha \cup \eta)=\nabla_{v} \alpha \cup \eta+\alpha \cup \nabla_{v} \eta
$$

But $\alpha \cup \eta=0$ because it is an element in $\mathscr{E}^{d, d-2}$.

3.2. The moduli space of hypersurfaces in $\mathbb{P}_{\Sigma}$. Let $\operatorname{Aut}_{\beta}\left(\mathbb{P}_{\Sigma}\right)$ be the subgroup of $\operatorname{Aut}\left(\mathbb{P}_{\Sigma}\right)$ which preserves the grading $\beta$. The coarse moduli space $\mathcal{M}_{\beta}$ for the general quasi-smooth hypersurfaces in $\mathbb{P}_{\Sigma}$ with divisor class $\beta$ may be constructed as a quotient

$$
U / \widetilde{\operatorname{Aut}}_{\beta}\left(\mathbb{P}_{\Sigma}\right)
$$

[3, 1], where $U$ is an open subset of $H^{0}\left(\mathbb{P}_{\Sigma}, \mathcal{O}_{\mathbb{P}_{\Sigma}}(X)\right)$, and $\widetilde{\operatorname{Aut}_{\beta}}\left(\mathbb{P}_{\Sigma}\right)$ is the unique nontrivial extension

$$
1 \rightarrow D(\Sigma) \rightarrow \widetilde{\operatorname{Aut}}_{\beta}\left(\mathbb{P}_{\Sigma}\right) \rightarrow \operatorname{Aut}_{\beta}\left(\mathbb{P}_{\Sigma}\right) \rightarrow 1
$$

By differentiating, we have a surjective map

$$
\kappa_{\beta}: H^{0}\left(\mathbb{P}_{\Sigma}, \mathcal{O}_{\mathbb{P}_{\Sigma}}(X)\right) \rightarrow T_{X} \mathcal{M}_{\beta},
$$

which is the analogue of the Kodaira-Spencer map.

The local system $\mathscr{H}^{d-1}$ and its various sub-systems do not descend to the moduli space $\mathcal{M}_{\beta}$, because the group $\operatorname{Aut}_{\beta}\left(\mathbb{P}_{\Sigma}\right)$ is not connected. Nevertheless, this group has a connected subgroup $\operatorname{Aut}_{\beta}^{0}\left(\mathbb{P}_{\Sigma}\right)$ of finite order, and, perhaps after suitably shrinking $U$, the quotient $\mathcal{M}_{\beta}^{0} \stackrel{\text { def }}{=} U / \operatorname{Aut}_{\beta}^{0}\left(\mathbb{P}_{\Sigma}\right)$ is a finite étale covering of $\mathcal{M}_{\beta}$ [7, 1].

Proposition 3.3. There is a morphism

$$
\gamma_{p}: T_{X} \mathcal{M}_{\beta} \otimes P H^{p, d-1-p}(X) \rightarrow P H^{p-1, d-p}(X)
$$

such that the diagram

$$
\begin{aligned}
H^{0}\left(\mathbb{P}_{\Sigma}, \mathcal{O}_{\mathbb{P}_{\Sigma}}(X)\right) \otimes H^{0}\left(\mathbb{P}_{\Sigma}, \Omega_{\mathbb{P}_{\Sigma}}^{d}((d-p) X)\right) & \stackrel{\cup}{\longrightarrow} H^{0}\left(\mathbb{P}_{\Sigma}, \Omega_{\mathbb{P}_{\Sigma}}^{d}((d-p+1) X)\right) \\
\kappa_{\beta} \otimes r_{p} \downarrow & r_{p-1} \downarrow \\
T_{X} \mathcal{M}_{\beta} \otimes P H^{p, d-1-p}(X) \longrightarrow & P H^{p-1, d-p}(X)
\end{aligned}
$$


commutes.

Proof. It suffices to prove the Proposition with $\mathcal{M}_{\beta}$ replaced by $\mathcal{M}_{\beta}^{0}$; in fact the tangent spaces at points $\mathcal{M}_{\beta}^{0}$ are canonically isomorphic to the tangent spaces at the image points in $\mathcal{M}_{\beta}$.

If $\rho: \mathcal{Z} \rightarrow \mathcal{M}_{\beta}^{0}$ is the induced map (where $\mathcal{Z}$ has been suitably restricted), the local system $\mathscr{H}^{d-1}$ descend to a local system $\rho_{*} \mathscr{H}^{d-1}$ on $\mathcal{M}_{\beta}^{0}$, and $\rho^{*} \rho_{*} \mathscr{H}^{d-1} \simeq$ $\mathscr{H}^{d-1}$ (the natural morphism $\mathscr{H}^{d-1} \rightarrow \rho^{*} \rho_{*} \mathscr{H}^{d-1}$ is an isomorphism on the stalks due to topological base change; note that $\rho$ is proper). Thus we obtain on $\mathcal{M}_{\beta}^{0}$ holomorphic bundles that are equipped with a Gauss-Manin connection, which is trivial in the direction of the fibers of $\rho$. If we define again $\gamma_{p}$ by $\gamma_{p}(v \otimes \alpha)=\nabla_{v}(\alpha)$ (where $\nabla$ is now the Gauss-Manin connection on $\mathcal{M}_{\beta}^{0}$ ), the commutavity of the diagram in the statement follows from the commutativity of the diagram (3).

The tangent space $T_{X} \mathcal{M}_{\beta}$ at a point representing a hypersurface $X$ is naturally isomorphic to the degree $\beta$ summand of the Jacobian ring of $f$, that is, $T_{X} \mathcal{M}_{\beta} \simeq$ $R(f)_{\beta}$ [3]. Moreover, by Proposition 2.12, $P H^{p, d-p-1}(X) \simeq R(f)_{(d-p) \beta-\beta_{0}}$.

Proposition 3.4. Under these isomorphisms, the morphism $\gamma_{p}$ in equation (6) coincides with the multiplication in the ring $R(f)$,

$$
R(f)_{\beta} \otimes R(f)_{(d-p) \beta-\beta_{0}} \rightarrow R(f)_{(d-p+1) \beta-\beta_{0}} .
$$

Proof. Theorem 9.7 in [3] implies

$$
H^{0}\left(\mathbb{P}_{\Sigma}, \Omega_{\mathbb{P}_{\Sigma}}^{d}((d-p) X) / H^{0}\left(\mathbb{P}_{\Sigma}, \Omega_{\mathbb{P}_{\Sigma}}^{d}((d-p-1) X) \simeq S_{(d-p) \beta-\beta_{0}},\right.\right.
$$

and, moreover, $H^{0}\left(\mathbb{P}_{\Sigma}, \mathcal{O}_{\mathbb{P}_{\Sigma}}(X)\right) \simeq S_{\beta}$; the cup product corresponds to the product in the ring $S$. This implies that the "top square" of the 3-dimensional diagram

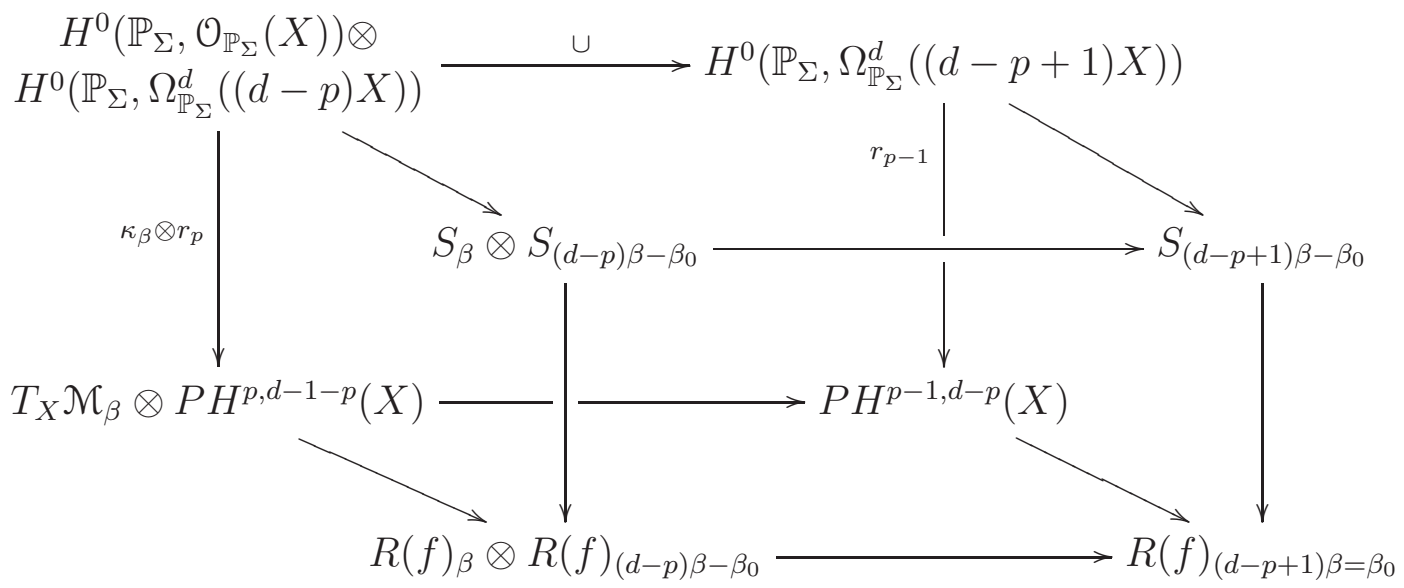


commutes. We need to show that the "bottom square" commutes as well, which will follow from the commutativity of the "side squares", and the surjectivity of the morphism $\kappa_{\beta} \otimes r_{p}$. The commutativity of the diagram on the right is contained in the proof of Theorem 10.6 in [3]. The commutativity of the diagram on the left follows from the commutativity of the previous diagram, with $d-p+1$ replaced by $d-p$, and the commutativity of

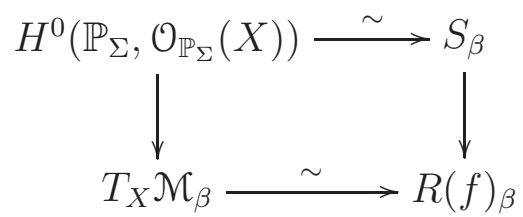

which is shown in the proof of Proposition 13.7 in [3].

3.3. Picard group. Our aim is now to prove the following result. Let us recall that a property is said to be very general if it holds in the complement of a countable union of subschemes of positive codimension [16]. Also recall that the Picard number $\rho(X)$ is the rank of the Néron-Severi group, i.e., $\rho(X)=\operatorname{dim}_{\mathbb{Q}}\left(H^{1,1}(X, \mathbb{C}) \cap\right.$ $\left.H^{2}(X, \mathbb{Q})\right)$.

Theorem 3.5. Let $\mathbb{P}_{\Sigma}$ be a 3-dimensional complete simplicial toric variety, $L$ an ample line bundle on $\mathbb{P}_{\Sigma}$, and $X$ a very general quasi-smooth hypersurface in the linear system $|L|$. If the morphism $\gamma_{2}: T_{X} \mathcal{M}_{\beta} \otimes P H^{2,0}(X) \rightarrow P H^{1,1}(X)$ is surjective, then $X$ and $\mathbb{P}_{\Sigma}$ have the same Picard number.

Theorem 3.5 will follow from two Lemmas. In the first Lemma no restriction on the dimension $d$ of $\mathbb{P}_{\Sigma}$ needs to be made, in the second we shall assume that $d$ is odd.

The first Lemma is an "infinitesimal Noether-Lefschetz theorem", such as Theorem 7.5.1 in [4].

Denote by $H_{T}^{d-1}(X) \subset H^{d-1}(X)$ the subspace of the cohomology classes that are annihilated by the action of the Gauss-Manin connection. Coefficients may be taken in $\mathbb{C}$ or $\mathbb{Q}$. Note that $H_{T}^{d-1}(X)$ has a Hodge structure.

Lemma 3.6. For a given $p$ with $1 \leq p \leq d-1$, assume that the morphism

$$
\gamma_{p}: T_{X} \mathcal{M}_{\beta} \otimes P H^{d-p, p-1}(X) \rightarrow P H^{d-p-1, p}(X)
$$

is surjective. Then $H_{T}^{p, d-1-p}(X)=i^{*}\left(H^{p, d-1-p}\left(\mathbb{P}_{\Sigma}\right)\right)$. 
Proof. Replace $\mathcal{M}_{\beta}$ by $\mathcal{M}_{\beta}^{0}$, and consider the local systems $\mathscr{E}^{d-1}$ and $\mathscr{P} \mathscr{E}^{p, d-p-1}$ on $\mathcal{M}_{\beta}^{0}$. Take

$$
\alpha \in H_{T}^{p, d-1-p}(X) \cap P H^{p, d-1-p}(X) .
$$

We regard classes in $P H^{p, d-1-p}(X)$ as elements in the fiber of $\mathscr{P} \mathscr{E}^{p, d-p-1}$ at the point $[X] \in M_{\beta}^{0}$. By hypothesis $\beta \in P H^{d-p-1, p}(X)$ can be written as $\beta=$ $\sum_{i} \gamma_{p}\left(t_{i} \otimes \eta_{i}\right)$ with $\eta_{i} \in P H^{d-p, p-1}(X)$. Then by equations (2) and (4)

$$
\langle\alpha, \beta\rangle=\sum_{i}\left\langle\alpha, \gamma_{p}\left(t_{i} \otimes \eta_{i}\right)\right\rangle=\sum_{i}\left\langle\alpha, \nabla_{t_{i}} \eta_{i}\right\rangle=-\sum_{i}\left\langle\nabla_{t_{i}} \alpha, \eta_{i}\right\rangle=0 .
$$

So $\alpha$ is orthogonal to $P H^{d-1-p, p}(X)$. By Lemma 2.8, this means that $\alpha$ is orthogonal to the whole group $H^{d-1-p, p}(X)$, hence it is zero. Therefore $H_{T}^{p, d-1-p}(X)=$ $i^{*}\left(H^{p, d-1-p}\left(\mathbb{P}_{\Sigma}\right)\right)$.

For any variety $Y$ we define $H^{m, m}(Y, \mathbb{Q})=H^{m, m}(Y, \mathbb{C}) \cap H^{2 m}(Y, \mathbb{Q})$.

Lemma 3.7. Let $d=2 m+1 \geq 3$, and assume that the hypotheses of the previous Lemma hold for $p=m$. Then for $z$ away from a countable union of subschemes of z of positive codimension one has

$$
H^{m, m}\left(X_{z}, \mathbb{Q}\right)=\operatorname{im}\left[i^{*}: H^{m, m}\left(\mathbb{P}_{\Sigma}, \mathbb{Q}\right) \rightarrow H^{2 m}\left(X_{z} \cdot \mathbb{Q}\right)\right] .
$$

Proof. Let $\bar{z}$ be the universal cover of $z$. On it the (pullback of the) local system $\mathscr{H}^{d-1}$ is trivial. Given a class $\alpha \in H^{m, m}(X)$ we can extend it to a global section of $\mathscr{H}^{d-1}$ by parallel transport using the Gauss-Manin connection. Define the subset $\bar{z}_{\alpha}$ of $\bar{z}$ as the common zero locus of the sections $\pi_{p}(\alpha)$ of $\mathscr{E}^{p, d-1-p}$ for $p \neq m$ (i.e., the locus where $\alpha$ is of type $(m, m))$.

If $\bar{z}_{\alpha}=\bar{z}$ we are done because $\alpha$ is in $H_{T}^{d-1}(X)$ hence is in the image of $i^{*}$ by the previous Lemma. If $\bar{z}_{\alpha} \neq \bar{z}$, we note that $\bar{z}_{\alpha}$ is a subscheme of $\bar{z}$.

We subtract from $z$ the union of the projections of the subschemes $\bar{z}_{\alpha}$ where $\bar{z}_{\alpha} \neq \bar{z}$. The set of these varieties is countable because we are considering rational classes.

Proof of Theorem 3.5. Lemma [3.7, for $d=3$, implies that $H^{1,1}\left(X_{z}, \mathbb{Q}\right)$ and $H^{1,1}\left(\mathbb{P}_{\Sigma}, \mathbb{Q}\right)$ have the same dimension for a very general $z$. These two numbers are the Picard numbers of $X_{z}$ and $\mathbb{P}_{\Sigma}$, respectively (see Definition/Proposition 2.2).

We assume now that $\mathbb{P}_{\Sigma}$ is Fano, and that $L=-K_{\mathbb{P}_{\Sigma}}$, so that the hypersurfaces in the linear system $|L|$ are K3 surfaces. We have $P H^{2,0} \simeq R(f)_{0} \simeq \mathbb{C}, P H^{1,1}(X) \simeq$ $R(f)_{\beta}$, and $T_{X} \mathcal{M}_{\beta} \simeq R(f)_{\beta}$, where $\beta=-\operatorname{deg} K_{\mathbb{P}_{\Sigma}}$. By Propositions 2.12 and 3.4, 
the morphism $\gamma_{2}$ corresponds to the multiplication $R(f)_{\beta} \otimes R(f)_{0} \rightarrow R(f)_{\beta}$, and since $R(f)_{0} \simeq \mathbb{C}$, this is an isomorphism. From Theorem 3.5 we have:

Theorem 3.8. Let $\mathbb{P}_{\Sigma}$ be a 3-dimensional Fano complete simplicial toric variety, and $X$ a very general hypersurface in the linear system $\left|-K_{\mathbb{P}_{\Sigma}}\right|$. Then $X$ has the same Picard number as $\mathbb{P}_{\Sigma}$.

\section{REFERENCES}

[1] P. S. Aspinwall, B. R. Greene, and D. R. Morrison, The monomial-divisor mirror map, Internat. Math. Res. Notices, (1993), pp. 319-337.

[2] V. V. BATYRev, Dual polyhedra and mirror symmetry for Calabi-Yau hypersurfaces in toric varieties, J. Algebraic Geom., 3 (1994), pp. 493-535.

[3] V. V. Batyrev and D. A. Cox, On the Hodge structure of projective hypersurfaces in toric varieties, Duke Math. J., 75 (1994), pp. 293-338.

[4] J. Carlson, S. Müller-Stach, and C. Peters, Period mappings and period domains, vol. 85 of Cambridge Studies in Advanced Mathematics, Cambridge University Press, Cambridge, 2003.

[5] D. A. Cox, Picard numbers of surfaces in 3-dimensional weighted projective spaces, Math. Z., 201 (1989), pp. 183-189.

[6] —- The homogeneous coordinate ring of a toric variety, J. Algebraic Geom., 4 (1995), pp. $17-50$.

[7] D. A. Cox, R. Donagi, And L. Tu, Variational Torelli implies generic Torelli, Invent. Math., 88 (1987), pp. 439-446.

[8] D. A. Cox And S. Katz, Mirror symmetry and algebraic geometry, vol. 68 of Mathematical Surveys and Monographs, American Mathematical Society, Providence, RI, 1999.

[9] V. I. Danilov, The geometry of toric varieties, Russian Math. Surveys, 33 (1978), pp. 97154.

[10] V. I. Danilov And A. G. KhovanskiĬ, Newton polyhedra and an algorithm for calculating Hodge-Deligne numbers, Izv. Akad. Nauk SSSR Ser. Mat., 50 (1986), pp. 925-945.

[11] A. J. De Jong And J. H. M. Steenbrink, Picard numbers of surfaces in 3-dimensional weighted projective spaces, Math. Z., 206 (1991), pp. 341-344.

[12] I. Dolgachev, Weighted projective varieties, in Group actions and vector fields (Vancouver, B.C., 1981), vol. 956 of Lecture Notes in Math., Springer, Berlin, 1982, pp. 34-71.

[13] M. Goresky and R. MacPherson, Stratified Morse theory, vol. 14 of Ergebnisse der Mathematik und ihrer Grenzgebiete (3), Springer-Verlag, Berlin, 1988.

[14] A. Klemm, D. Maulik, R. Pandharipande, and E. Scheidegger, Noether-Lefschetz theory and the Yau-Zaslow conjecture, J. Amer. Math. Soc., 23 (2010), pp. 1013-1040.

[15] R. Kloosterman, Higher Noether-Lefschetz loci of elliptic surfaces, J. Differential Geom., 76 (2007), pp. 293-316. 
[16] R. Lazarsfeld, Positivity in algebraic geometry. I, vol. 48 of Ergebnisse der Mathematik und ihrer Grenzgebiete (3), Springer-Verlag, Berlin, 2004. Classical setting: line bundles and linear series.

[17] D. Maulik, R. Pandharipande, and A. Pixton, Curves on K3 surfaces and modular forms. arXiv:1001.2719 [math.AG].

[18] G. V. Ravindra And V. SRInivas, The Grothendieck-Lefschetz theorem for normal projective varieties, J. Algebraic Geom., 15 (2006), pp. 563-590.

[19] S.-S. RoAn, Picard groups of hypersurfaces in toric varieties, Publ. Res. Inst. Math. Sci., 32 (1996), pp. 797-834.

[20] F. RohsiePe, Lattice polarized toric K3 surfaces. arXiv:hep-th/0409290v1.

[21] M. Saito, Mixed Hodge modules, Publ. Res. Inst. Math. Sci., 26 (1990), pp. 221-333.

[22] I. Satake, On a generalization of the notion of manifold, Proc. Nat. Acad. Sci. U.S.A., 42 (1956), pp. 359-363.

[23] J. Steenbrink, Intersection form for quasi-homogeneous singularities, Compositio Math., 34 (1977), pp. 211-223.

[24] — On the Picard group of certain smooth surfaces in weighted projective spaces, in Algebraic geometry (La Rábida, 1981), vol. 961 of Lecture Notes in Math., Springer, Berlin, 1982, pp. 302-313.

[25] L. TU, Macaulay's theorem and local Torelli for weighted hypersurfaces, Compositio Math., 60 (1986), pp. 33-44.

[26] Z. Z. Wang And D. Zaffran, A remark on the hard Lefschetz theorem for Kähler orbifolds, Proc. Amer. Math. Soc., 137 (2009), pp. 2497-2501. 